\title{
Transmission Experiment of Bandwidth Compressed Carrier Aggregation in a Realistic Fading Channel
}

\author{
Tongyang Xu, Student Member, IEEE and Izzat Darwazeh, Senior Member, IEEE
}

\begin{abstract}
In this paper, an experimental testbed is designed to evaluate the performance of a bandwidth compressed multicarrier technique termed spectrally efficient frequency division multiplexing (SEFDM) in a carrier aggregation (CA) scenario ${ }^{1}$. Unlike orthogonal frequency division multiplexing (OFDM), SEFDM is a non-orthogonal waveform which, relative to OFDM, packs more sub-carriers in a given bandwidth, thereby improving spectral efficiency. $\mathrm{CA}$ is a long term evolution-advanced (LTE-Advanced) featured technique that offers a higher throughput by aggregating multiple legacy radio bands. Considering the scarcity of radio spectrum, SEFDM signals can be utilized to enhance CA performance. The combination of the two techniques results in a larger number of aggregated component carriers (CCs) and therefore increased data rate in a given bandwidth with no additional spectral allocation. It is experimentally shown that CA-SEFDM can aggregate up to $7 \mathrm{CCs}$ in a limited bandwidth while CA-OFDM can only put $5 \mathrm{CCs}$ in the same bandwidth. In this work, LTE-like framed CA-SEFDM signals are generated and delivered through a realistic LTE channel. A complete experimental setup is described together with error performance and effective spectral efficiency comparisons. Experimental results show that the measured BER performance for CA-SEFDM is very close to CA-OFDM and the effective spectral efficiency of CA-SEFDM can be substantially higher than that of CA-OFDM.
\end{abstract}

Index Terms-Multicarrier communications, carrier aggregation, 5G, spectral efficiency, OFDM, SEFDM, non-orthogonal.

\section{INTRODUCTION}

$\mathbf{T}$ HE exponential growth in demand for broadband services is leading to increased research in new modulation formats and system architectures aiming to utilize the available, yet limited, spectrum. Long term evolution (LTE) [2] was proposed and standardized and has now been commercialized, with LTE-Advanced [3] being introduced and its high data rate features demonstrated. Among these techniques, carrier aggregation (CA) [4][5][6][7] is one of the most attractive and distinct. The main idea of this technique is to aggregate legacy fragmented frequency bands (i.e. LTE signal band) up to 100 $\mathrm{MHz}$. However, this benefit is subject to spectrum availability. Orthogonal frequency division multiplexing (OFDM) has been

Copyright (c) 2015 IEEE. Personal use of this material is permitted. However, permission to use this material for any other purposes must be obtained from the IEEE by sending a request to pubs-permissions@ieee.org.

T. Xu and I. Darwazeh are with the Department of Electronic and Electrical Engineering, University College London, London, WC1E 7JE, UK (e-mail: t.xu@ee.ucl.ac.uk, i.darwazeh@ucl.ac.uk).

${ }^{1}$ This journal submission is an extension of the authors' recent conference paper [1], which was the first to propose the use of SEFDM in an LTE framework. This extended paper details SEFDM signal generation and detection methods, channel estimation techniques and their efficacy as well as complexity analysis of different SEFDM variants. identified as a physical layer air interface in LTE. OFDM is the multicarrier technique which can pack multiple overlapping orthogonal sub-carriers. However, OFDM's transmission bandwidth is close to that achieved using a single carriers and OFDM is sensitive to frequency offset, which results in performance degradation. In order to relax the requirement of orthogonality and simultaneously achieve a higher spectral efficiency, spectrally efficient frequency division multiplexing (SEFDM) was proposed in 2003 [8]. SEFDM introduces non-orthogonal overlapping sub-carriers leading to an improved spectral efficiency. This technique was proposed for $5^{\text {th }}$ generation $(5 \mathrm{G})$ networks [9] and has been experimentally implemented in optical [10][11], wireless [1] and hybrid [12] systems. This and other promising spectrally efficient techniques proposed for future $5 \mathrm{G}$ have been recently detailed in a new book [13] and some were discussed in [14]. Some of these techniques are based on non-orthogonal signal formats and these have been eloquently summarised in a 2013 IEEE proceedings paper with the title "Faster-Than-Nyquist Signaling"[15]. FTN itself is a method applicable to single carrier and multicarrier systems and was originally proposed by Mazo in 1975 [16]. The FTN method was first proposed for multicarrier systems initially in 2005 [17] and further detailed in [18] with transmitter and receiver hardware implementation in [19]. Multistream FTN is a time-domain non-orthogonal technique offering improved spectral efficiency. Instead of packing sub-carriers closer together as in SEFDM, it transmits higher rate data on the subcarriers, thereby violating the Nyquist criteria and leading to a non-orthogonal multicarrier signal. Furthermore, a hybrid technique termed time frequency packing (TFP), which may be viewed as a combination of SEFDM and FTN was presented in [20]. The time domain nature of the FTN and TFP techniques makes it difficult to place their respective signals in LTE frames, which have strict timing requirements. A group of pulse shaping techniques, such as filterbank based multicarrier (FBMC) technique [21], generalized frequency division multiplexing (GFDM) technique [22] and universal-filtered multi-carrier (UFMC) technique [23] were proposed to reduce interference power by removing out-of-band emission. These signal shaping/filtering techniques, although have the desirable characteristic of almost brick wall signal spectra, they offer no bandwidth saving or data rate increase within the signal band. SEFDM, on the other hand, offers both advantages; signals that may be placed within LTE frames with minimal modification, hence good LTE compatibility and higher data rate through spectral saving. Furthermore,recent studies of SEFDM have shown its capacity merits [24] and similarly to FBMC, GFDM and UFMC techniques, its reduction of out of band emission 
[25][26] advantages when the SEFDM pulses are shaped by filtering at the transmitter. Given these signal advantages, we believe the use of SEFDM in future wireless systems, such as $5 \mathrm{G}$ ones which require higher levels of spectral efficiency and LTE backward compatibility, is a credible proposal.

Signal detection is challenging due to the self-created inter carrier interference (ICI) in SEFDM. Initially, linear detectors were evaluated such as zero forcing (ZF) [8] and minimum mean squared error (MMSE) [27]. However, they perform well only for small system sizes (i.e. small number of subcarriers) and at high SNRs. Maximum likelihood (ML) is an optimal detection technique to recover signals subject to ICI, but it has a major drawback in the exponential growth of its complexity with the enlargement of the system size and modulation level. Sphere decoding (SD), an alternative to $\mathrm{ML}$, was demonstrated in [28] to have an optimal performance at a much reduced complexity by searching candidate solutions within a constrained space. A key limitation is in its random complexity, which depends on noise levels and leads to impractical hardware implementation. Subsequently, a hybrid detector combining truncated singular value decomposition (TSVD) with fixed sphere decoding (FSD) was explored in [27], which offered a substantial reduction in complexity. However, the achievable error performance is still worse than the optimum ML performance. In order to improve further the system performance, a hybrid iterative detectionFSD (ID-FSD) technique was reported in [29] where 20\% of the bandwidth is saved without performance degradation. Unfortunately, the aforementioned detectors are all limited to small system sizes because as the number of sub-carriers is increased, the elimination of ICI at the receiver becomes increasingly difficult. To ameliorate this problem, work in [30] proposed an efficient SEFDM system termed block-spectrally efficient frequency division multiplexing (B-SEFDM) where the whole spectrum is decomposed into several sub-bands and symbols in each sub-band can be recovered independently. Due to a limited number of sub-carriers within each sub-band, B-SEFDM can effectively remove out-of-band interference and employ optimal detection algorithms like ML or SD in each sub-band with correspondingly reduced complexity. Results show that by using SD, an SEFDM signal of $20 \%$ bandwidth saving with 128 sub-carriers can be recovered with no performance degradation. In B-SEFDM the complexity is linearly proportional to the number of sub-bands since identical detectors are used in each sub-band. To reduce complexity further and maintain (or even improve) error performance, it is important to use different methods of transmission and detection. This challenge was addressed recently in [31] by using a convolutional coding assisted soft detector to improve iteratively system performance. A soft detector consisting of an FFT detector followed by a standard BCJR decoder [32] was designed to allow soft information to be exchanged between the detector and the decoder for the purpose of improving the reliability of candidate solutions in each iteration. This detector is a practical solution for a large size SEFDM system due to the introduction of the FFT detector in which an efficient FFT algorithm is employed. Results of [31] reported that in a frequency selective channel scenario, an SEFDM system with 1024 non-orthogonal sub-carriers can save at least $40 \%$ bandwidth compared to an equivalent OFDM system, with a $1.1 \mathrm{~dB}$ performance penalty. It is worth noting that SEFDM has been studied in different research directions. Work in [33] investigated the effect of nonlinear distortions caused by power amplifiers on SEFDM. The authors of [25] found that the bandwidth compression issue in SEFDM can be optimized using signals with optimum envelope forms, which have low out-of-band power emission. Moreover, work in [34] proved that an asymptotically optimal algorithm initially proposed for OFDM signal reception can be used for SEFDM.

In this work, we consider respective advantages of both CA and SEFDM in a real RF environment, which introduces amplitude attenuation, phase distortion and propagation delay. Recalling that $\mathrm{CA}$ is a bandwidth extension scheme while SEFDM is a bandwidth compression technique, the combination of the two results in more aggregated CCs in a given bandwidth. This can result in two possible scenarios; either higher throughput may be achieved without occupying additional bandwidth or more users (more CCs) can share the same overall bandwidth. In this paper the use of SEFDM in a CA scenario is discussed conceptually and then evaluated experimentally in a realistic RF scenario. An LTE-like CASEFDM experimental testbed is demonstrated where more CCs are aggregated in a given LTE-Advanced standard $25 \mathrm{MHz}$ bandwidth. Experimentally, signals were generated using a commercially available arbitrary waveform generator (AWG), transmitted through an LTE fading channel emulator and then digitized and recovered. Signals were based on LTE signal format with modification of the placing of pilot tones to facilitate channel estimation in the heavily interfered SEFDM signals. The recovery included full channel estimation and equalization before signal demodulation and detection.

The rest of the paper is organized as follows. Section II gives a brief description of the principle of CA-SEFDM system. Section III introduces the SEFDM system model, and presents the key digital signal processing including signal generation, channel estimation and signal detection. Section IV describes the experimental setup for the CA-SEFDM transceiver with a realistic fading channel and Section $\mathrm{V}$ shows the measured results from the experimental testbed. Implementation challenges are demonstrated in Section VI. Finally, Section VII concludes the paper.

\section{PRINCIPLE OF CA-SEFDM}

LTE-Advanced allows several carrier aggregation scenarios to increase the system bandwidth beyond $20 \mathrm{MHz}$ [3]. CCs can be aggregated contiguously in the same band (intra-band contiguous CA), non-contiguously in the same band (intraband non-contiguous $\mathrm{CA}$ ), or in different bands (inter-band non-contiguous CA). From a digital signal processing perspective, there is no obvious difference among these scenarios. However, from an RF implementation perspective [5][6][7], the intra-band contiguous CA is the simplest, where a single RF filter and a single IFFT/FFT can be used to reduce implementation complexity, at the expense of using a wider band RF filter. For non-contiguous CA scenarios, either in 


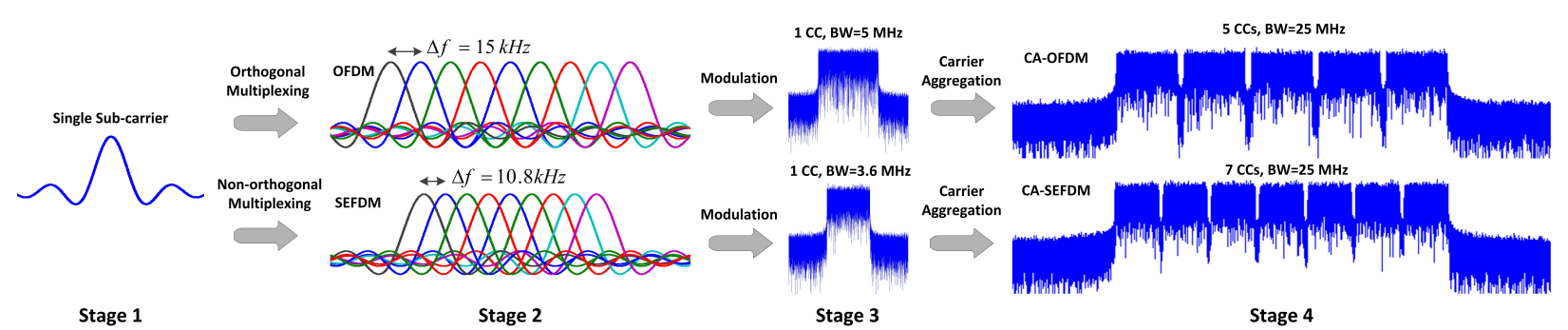

Figure 1. Extension to wider channel bandwidth by means of carrier aggregation for both OFDM and SEFDM. CC indicates component carrier. BW is the channel bandwidth including data bandwidth and $10 \%$ protection bandwidth.

the same band or different bands, several RF filters and IFFTs/FFTs have to be used. In this work we employ the intra-band contiguous CA scheme. The stage where CCs are combined leads to alternative architectures [35]. In this work, a simple CA transmitter whose multiple CCs are combined in the digital domain before digital-to-analogue conversion (DAC) is used according to [35]. Thus, the signal generation is simplified using a single IFFT.

The general CA-SEFDM idea is illustrated in Fig. 1. A single sub-carrier, with $15 \mathrm{kHz}$ baseband bandwidth, is generated. In the figure, both OFDM and SEFDM sub-carrier packing schemes are demonstrated for the purpose of comparison. For OFDM orthogonal multiplexing, multiple sub-carriers are orthogonally packed at each frequency with $15 \mathrm{kHz}$ sub-carrier spacing. For SEFDM, after non-orthogonal multiplexing, subcarriers are packed more densely, thus the spacing between adjacent sub-carriers is smaller than $15 \mathrm{kHz}$ (i.e. below the orthogonality limit). It is apparent that by multiplexing the same number of sub-carriers, SEFDM will occupy less bandwidth. In the figure, the signal spectra of OFDM and SEFDM CCs are illustrated showing bandwidth compression in SEFDM CCs and the aggregation of higher number of CCs, with narrower guard bands, in CA-SEFDM whilst maintaining the same data rate per sub-carrier. Therefore, for the same bandwidth allocation, the benefits of CA-SEFDM over CAOFDM are evident. CA-SEFDM can offer a higher throughput for a single user. Alternatively, more users (more CCs) can share the same bandwidth in the CA-SEFDM.

\section{SEFDM SIGNAL}

An SEFDM symbol consists of a block of $N$ complex symbols, denoted by $s=s_{\Re}+j s_{\Im}$. These complex symbols are modulated on non-orthogonal overlapped sub-carriers. Therefore, for a system with $N$ sub-carriers, the normalized SEFDM signal is expressed as

$$
x(t)=\frac{1}{\sqrt{T}} \sum_{l=-\infty}^{\infty} \sum_{n=0}^{N-1} s_{l, n} \exp \left(\frac{j 2 \pi n \alpha(t-l T)}{T}\right)
$$

where $\alpha=\Delta f T$ is the bandwidth compression factor, where $\Delta f$ denotes the sub-carrier spacing and $T$ is the period of one SEFDM symbol. $N$ is the number of sub-carriers and $s_{l, n}$ is the complex QAM symbol modulated on the $n^{\text {th }}$ sub-carrier in the $l^{\text {th }}$ SEFDM symbol. Since $\alpha$ defines the bandwidth compression, then the percentage of bandwidth saving is equal to $(1-\alpha) \times 100 \%$. Notice that $\Delta f$ in SEFDM is smaller than that in OFDM. For OFDM signals $\alpha=1$, and $\alpha<1$ for SEFDM.

In this work, we focus on the discrete sampled version where the first SEFDM symbol of $x(t)$ is sampled at $T / Q$ intervals where $Q=\rho N$ and $\rho \geq 1$ is the oversampling factor. Hence, the discrete SEFDM signal is mathematically represented by

$$
X[k]=\frac{1}{\sqrt{Q}} \sum_{n=0}^{N-1} s_{n} \exp \left(\frac{j 2 \pi n k \alpha}{Q}\right)
$$

where $X[k]$ is the $k^{\text {th }}$ time sample of $x(t)$ where $k=$ $[0,1, \ldots, Q-1], s_{n}$ is a QAM symbol modulated on the $n^{t h}$ sub-carrier and $\frac{1}{\sqrt{Q}}$ is a scaling factor for the purpose of normalization. Furthermore, the signal can be simply expressed in matrix form as:

$$
X=\mathbf{F} S
$$

where $X$ is a $Q$-dimensional vector of time samples, $S$ is an $N$-dimensional vector of transmitted symbols and $\mathbf{F}$ is a $Q \times N$ sub-carrier matrix with elements equal to $e^{\frac{j 2 \pi n k \alpha}{Q}}$.

Assume a simple channel scenario where only AWGN is introduced. At the receiver, $X$ defined in (3) is contaminated by additive white Gaussian noise (AWGN) denoted as $Z$. The received signal is demodulated by correlating it with the conjugate sub-carriers $\mathbf{F}^{*}$. The reception process is expressed as

$$
R=\mathbf{F}^{*} X+\mathbf{F}^{*} Z=\mathbf{F}^{*} \mathbf{F} S+\mathbf{F}^{*} Z=\mathbf{C} S+Z_{\mathbf{F}^{*}}
$$

where $R$ is an $N$-dimensional vector of demodulated symbols or in other words collected statistics, $\mathbf{C}$ is an $N \times N$ correlation matrix which is defined as $\mathbf{C}=\mathbf{F}^{*} \mathbf{F}$, where $\mathbf{F}^{*}$ denotes the $N \times Q$ conjugate sub-carrier matrix with elements equal to $e^{\frac{-j 2 \pi n k \alpha}{Q}}$ for $k=[0,1, \ldots, Q-1]$ and $Z_{\mathbf{F}^{*}}$ is the AWGN correlated with the conjugate sub-carriers. Interference from non-orthogonal packed sub-carriers can be evaluated using the correlation matrix $\mathbf{C}$ where elements in the matrix are expressed as 


$$
\begin{aligned}
c_{m, n} & =\frac{1}{Q} \sum_{k=0}^{Q-1} e^{\frac{j 2 \pi m k \alpha}{Q}} e^{-\frac{j 2 \pi n k \alpha}{Q}} \\
& =\frac{1}{Q} \times\left\{\begin{array}{ll}
Q & , m=n \\
\frac{1-e^{j 2 \pi \alpha(m-n)}}{1-e^{\frac{j 2 \pi(m-n)}{Q}}} & , m \neq n
\end{array}\right\}
\end{aligned}
$$

where $m, n$ are indices of two arbitrary sub-carriers. The offdiagonal terms in the correlation matrix $\mathbf{C}$ indicate the effect of non-orthogonal overlapping, which results in ICI.

\section{A. Signal Generation}

In a conventional OFDM system, signal generation can be realized in a straightforward manner by using a standard inverse fast Fourier transform (IFFT). However, due to the deliberate violation of the orthogonality property within SEFDM, the traditional IFFT approach is not applicable and therefore the direct application of (2) to effect signal generation is not an option. In order to use the IFFT algorithm, two alternative algorithms were specially designed for SEFDM. Either using a single IFFT or multiple IFFTs [36]. The above methods are also applicable to SEFDM signal demodulation since the FFT is an inverse operation of the IFFT.

1) Single IFFT: The first method is a zero padding scheme where a specific number of zeros are padded at the end of each input vector (i.e. multiple QAM symbols). The length of an original input vector becomes $M=Q / \alpha$. Therefore, for an $N$ sub-carrier SEFDM system with the bandwidth compression factor $\alpha$, it is possible to employ a single IFFT of a $Q / \alpha$ length where useful symbols are appended to the first $N$ inputs and zeros are appended to the last $M-N$ remaining inputs such as

$$
s_{i}^{\prime}= \begin{cases}s_{i} & 0 \leq i<N \\ 0 & N \leq i<M\end{cases}
$$

where the value of $Q / \alpha$ has to be an integer and simultaneously a power of two, in other words $Q / \alpha \in 2^{\left(\mathbb{N}_{>0}\right)}$, allowing for the IDFT to be implemented by means of the computationally efficient radix-2 IFFT. Assuming $Q / \alpha$ satisfies the requirement, the SEFDM signal in a new format is expressed as

$$
X^{\prime}[k]=\frac{1}{\sqrt{M}} \sum_{n=0}^{M-1} s_{n}^{\prime} \exp \left(\frac{j 2 \pi n k}{M}\right)
$$

where $n, k=[0,1, \ldots, M-1]$. The output is truncated with only $Q$ samples retained while the rest of the samples are discarded.

2) Multiple IFFTs: Work in [36] presents a technique to effectively generate SEFDM signals by using a multiple IFFT architecture, which relaxes the requirement for $Q$ and $\alpha$. Instead of padding zeros at the end of each input symbol, a number of zeros are interpolated. It is shown in [36] that by setting $\alpha=b / c$, where $b$ and $c$ are both positive integers (i.e. $\left.b, c \in \mathbb{N}_{>0}\right)$ and $b<c$, the rearranged input vector of length $c Q$ can be separated into $c$ parallel vectors where an IFFT of length $Q$ can be operated on each vector. Therefore, (2) can be rearranged and represented as

$$
X[k]=\frac{1}{\sqrt{Q}} \sum_{n=0}^{c Q-1} s^{\prime}(n) \exp \left(\frac{j 2 \pi n k}{c Q}\right)
$$

where $s^{\prime}$ is a cQ-dimensional vector of symbols as

$$
s^{\prime}(i)=\left\{\begin{array}{ll}
s_{i / b} & i \bmod b=0 \\
0 & \text { otherwise }
\end{array} .\right.
$$

By substituting with $n=i+l c$, (8) can be extended to

$$
X[k]=\frac{1}{\sqrt{Q}} \sum_{i=0}^{c-1} \sum_{l=0}^{Q-1} s^{\prime}(i+l c) \exp \left(\frac{j 2 \pi k(i+l c)}{c Q}\right) .
$$

With further modifications, (10) can be rearranged as

$$
X[k]=\frac{1}{\sqrt{Q}} \sum_{i=0}^{c-1} \exp \left(\frac{j 2 \pi i k}{c Q}\right) \sum_{l=0}^{Q-1} s^{\prime}(i+l c) \exp \left(\frac{j 2 \pi l k}{Q}\right) .
$$

Equation (11) shows that an SEFDM symbol is equivalent to a combination of multiple OFDM symbols. It is apparent that the SEFDM signal can be generated by using $c$ parallel IFFT operations each of $Q$ points. The first summation term on the right hand side of (11) determines the number of parallel IFFT operations. The second summation term indicates a Q-point IFFT of the sequence $s^{\prime}(i+l c)$.

Much work has been done with respect to hardware implementations of the multiple IFFT method. A route to very large scale integration (VLSI) implementation of a reconfigurable SEFDM transmitter in 32-nm CMOS was published in [36] with the introduction of a pruned IFFT architecture. At 100 $\mathrm{MHz}$ clock frequency, the throughput can reach 17.4 Mbps with QPSK modulation, and up to 52.2 Mbps with 64QAM modulation, with bandwidth saving up to $50 \%$ relative to OFDM.

\section{B. Channel Estimation}

In OFDM systems a single tap frequency-domain channel estimation algorithm may be applied to estimate the time varying and frequency selective channel. Alas, this is not possible for SEFDM due to the self-created ICI. Some related work [37][38] has been done to resolve this issue present in SEFDM. A time-domain channel estimation and a frequencydomain joint channel equalization/detection were proposed in [37] where an SEFDM pilot symbol is used to estimate channel state information (CSI), which is termed full channel estimation (FCE). However, due to non-orthogonal packing of sub-carriers in SEFDM, pilot tones (reference signals In LTE terminology) within one SEFDM pilot symbol interfere with each other leading to inaccurate estimate of CSI. In [38], an enhanced time-domain estimation/equalization method, termed partial channel estimation (PCE), was investigated. This was based on the analysis in [38], where for specific values of $\alpha$, there exists a number of non-adjacent sub-carriers that are mutually orthogonal. Therefore, pre-determined pilot tones can be sent on such mutually orthogonal sub-carriers to avoid ICI. Although, other mutually non-orthogonal sub-carriers 
are reserved without sending data, a more accurate channel estimate can be obtained.

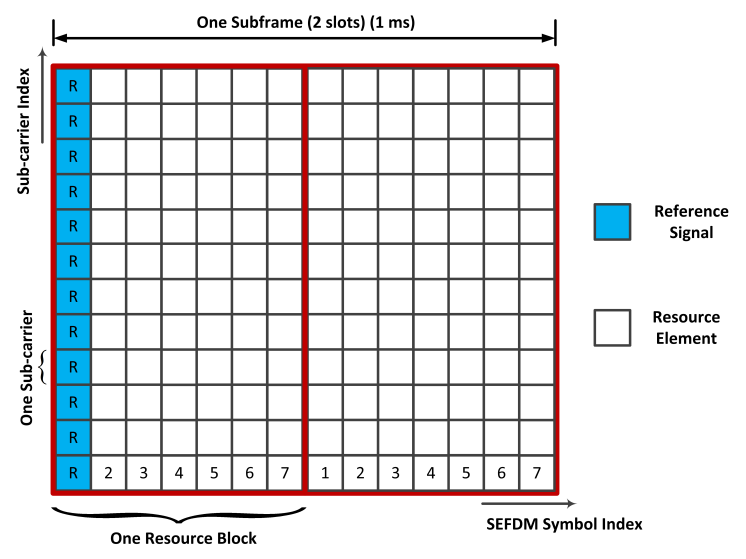

Figure 2. SEFDM resource block definition and location of pilot tones (reference signals) for a single antenna system. One resource element indicates one 4QAM symbol modulated on one sub-carrier at one time instant. Each resource block consists of 12 sub-carriers (i.e. $180 \mathrm{kHz}$ ) and 7 SEFDM symbols.

In this work, we adopt the FCE to estimate CSI and equalize the channel in the time-domain, which indicates pilot tones are modulated on all sub-carriers instead of the mutually orthogonal ones. A purpose designed SEFDM subframe is illustrated in Fig. 2, with data structure identical to that of an LTE subframe [2] except the location of pilot symbols. For an SEFDM subframe, the first SEFDM symbol is reserved as the pilot symbol instead of reference signals distributed within one LTE subframe. The CSI can be obtained at the receiver based on the knowledge of the pilot symbol. In order to get more accurate CSI, the estimated values of CSI are averaged from two pilot symbols of adjacent subframes. It is worth noting that the SEFDM symbols in two resource blocks within the same subframe $(1 \mathrm{~ms})$ are assumed to experience the same channel.

The concept of SEFDM time-domain channel estimation/equalization is theoretically described in [38] and practically tested in [1][12]. In order to evaluate the efficacy of the time-domain channel estimation scheme, a static frequency selective channel [31] as shown in (12) is assumed.

$$
\begin{aligned}
h(t)= & 0.8765 \delta(t)-0.2279 \delta\left(t-T_{s}\right)+0.1315 \delta\left(t-4 T_{s}\right) \\
& -0.4032 e^{\frac{j \pi}{2}} \delta\left(t-7 T_{s}\right) .
\end{aligned}
$$

The estimation accuracy of different channel estimation methods is measured using mean squared error (MSE) as shown in Fig. 3. The MSE is calculated by

$$
M S E=\frac{1}{n} \sum_{i=0}^{n-1}\left(\hat{h}_{i}-h_{i}\right)^{2} .
$$

Results are compared to those of a frequency-domain channel estimator similar to that used in OFDM systems. For the frequency-domain channel estimation, expectedly the OFDM system results in better channel parameter estimates

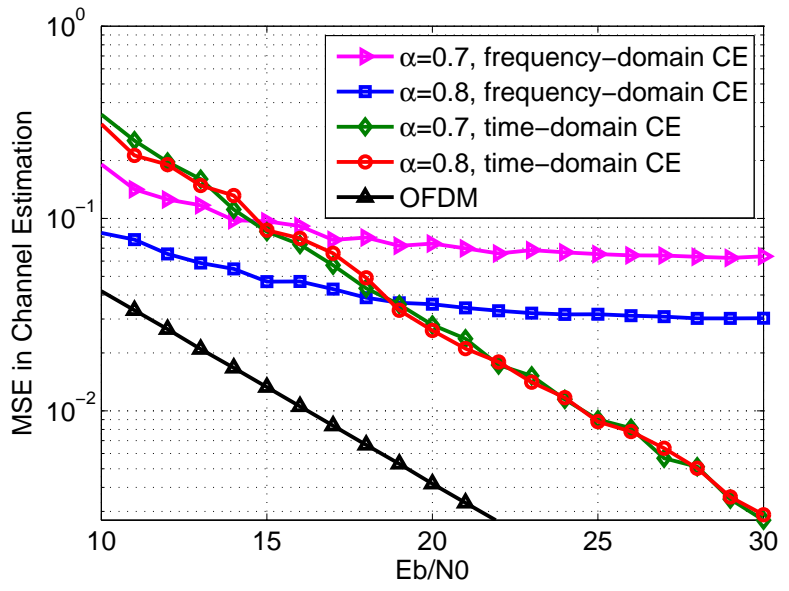

Figure 3. MSE of time-domain and frequency-domain channel estimation methods. FFT size is 128 and 9 samples are used as CP.

and therefore has a lower MSE values than those of the SEFDM system tested for different values of $\alpha$. This illustrates that the loss of orthogonality in the SEFDM system impairs the performance of the commonly used frequency-domain single tap channel estimation. It is evident that, with the increase of $\alpha$, the SEFDM result approaches those of OFDM. Conversely, for the time-domain channel estimation, although the MSE values are still worse for SEFDM relative to those of OFDM, variation of $\alpha$ results in minimal variation of MSE values. It should be noted that at low $E_{b} / N_{o}$, the frequency-domain method provides a more accurate channel estimation than the time-domain one. At high $E_{b} / N_{o}$, since the typical frequency-domain method cannot effectively remove ICI, an error floor occurs. On the other hand, the time-domain method can mitigate the ICI effect, therefore, the time-domain method shows a lower MSE than the frequency-domain one. In addition, it is apparent that the OFDM system shows a lower MSE than the proposed SEFDM channel estimation scheme. The reason is attributed to the ill conditioning (i.e. self-created ICI as shown in (5)) of the SEFDM system.

\section{SEFDM Soft Detection Principle}

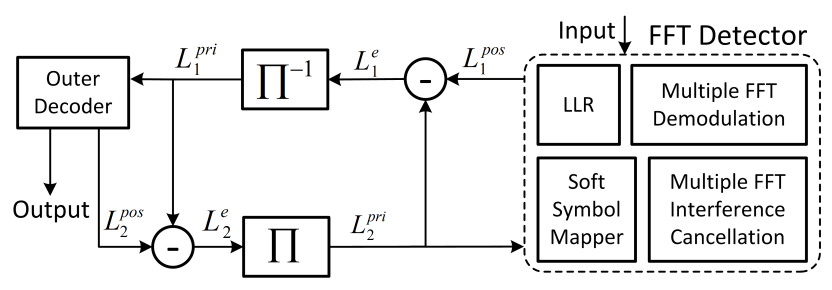

Figure 4. Functional block diagram of the Turbo-SEFDM soft detector.

The utility of applying soft detection in SEFDM signals operating in different multipath environments was reported recently in [1] and the soft detection principle was described in [31]. Similar techniques were used for FTN [19][39] while TFP has successfully applied the low density parity check 
(LDPC) with interference cancellation in [20]. In this section the principle is summarised and readers are referred to the above mentioned references for further details. A functional block diagram of the soft detector is illustrated in Fig. 4. In this figure, the soft detector consists of an FFT detector [31] and an outer decoder [32]. The proposed architecture maximizes the a posteriori probability (APP) for a given bit through a process of iteration based on the Turbo principle [40]. In each iteration, soft (i.e. extrinsic) information $L^{e}$ is exchanged between the FFT based detector and the outer decoder. The soft information is expressed in the form of log-likelihood ratio (LLR). The sign of the LLR value determines the sign of the bit, and its magnitude determines the reliability of the sign of the bit. The extrinsic information $L^{e}$ is obtained by subtracting a priori information from a posteriori information written as $L^{e}=L_{a-\text { posterior } i}-L_{a-\text { prior } i}$. In Fig. 4 , at the beginning, the input samples are demodulated in the multiple FFT demodulator. Then, the a posteriori information $L_{1}^{\text {pos }}$ is generated from the LLR module inside the FFT detector and is transformed to extrinsic information $L_{1}^{e}$ by subtracting a priori information $L_{2}^{p r i}$. This extrinsic information is deinterleaved and delivered to the outer decoder as the a priori information $L_{1}^{p r i}$. The outer decoder outputs a posteriori information $L_{2}^{\text {pos }}$ which then generates extrinsic information $L_{2}^{e}$. This information is interleaved and sent back to the soft symbol mapper within the FFT detector. Updated soft QAM symbols are fed to the multiple FFT/IFFT interference cancellation module for the purpose of removing interference.

The study of the outer decoder is beyond the scope of this work. Detailed description of the standard BCJR decoder can be found in [32]. The demodulation is an inverse operation of the modulation in (2). In order to use FFT for SEFDM signal demodulation, following the same principle in section III-A2, the demodulation of the SEFDM signal can be treated as a manipulation of $c$ parallel overlapped OFDM signals expressed as

$R[n]=\frac{1}{\sqrt{Q}} \sum_{i=0}^{c-1} \exp \left(\frac{-j 2 \pi n i}{c Q}\right) \sum_{l=0}^{Q-1} r^{\prime}(i+l c) \exp \left(\frac{-j 2 \pi n l}{Q}\right)$

where $r$ is the received sample vector and $r^{\prime}$ is a $c Q$ dimensional vector of samples as

$$
r^{\prime}(i)=\left\{\begin{array}{ll}
r_{i / b} & i \bmod b=0 \\
0 & \text { otherwise }
\end{array} .\right.
$$

The second summation term in (14) is a Q-point FFT of the sequence $r^{\prime}(i+l c)$. Considering the first term, the demodulation of an SEFDM signal can be treated as multiple FFT operations indicating one SEFDM symbol is composed of multiple overlapped OFDM symbols. It is apparent that one OFDM symbol is an interference signal superimposed on other OFDM symbols. Therefore, the interference canceller aims to remove $c-1$ parallel OFDM interference signals from one OFDM signal, through regenerating interference signals using IFFT processes applied to the soft mapped symbols. Detailed mathematical descriptions of these processes can be found in [31].
Since a Turbo principle architecture is employed, the interference canceller and demodulator are not activated simultaneously. Since an FFT design can be easily transformed to an IFFT by carrying out extra computations (such as conjugating input complex QAM symbols and output complex results), there is an implementation advantage of this architecture in that the FFT detector can reuse the demodulation and the interference cancellation functions by applying appropriate time multiplexing.

\section{EXPERIMENTAL SetuP}

This section presents experimental evaluation of CASEFDM tested in an LTE wireless fading channel scenario. The experimental testbed of CA-SEFDM is shown in Fig. 5. The testbed includes hardware and software elements. The software consists of two DSP blocks for signal generation and detection at the transmitter and the receiver, respectively. The hardware consists of an Aeroflex PCI extensions for instrumentation (PXI) chassis, a Spirent VR5 channel emulator and a spectrum analyzer.

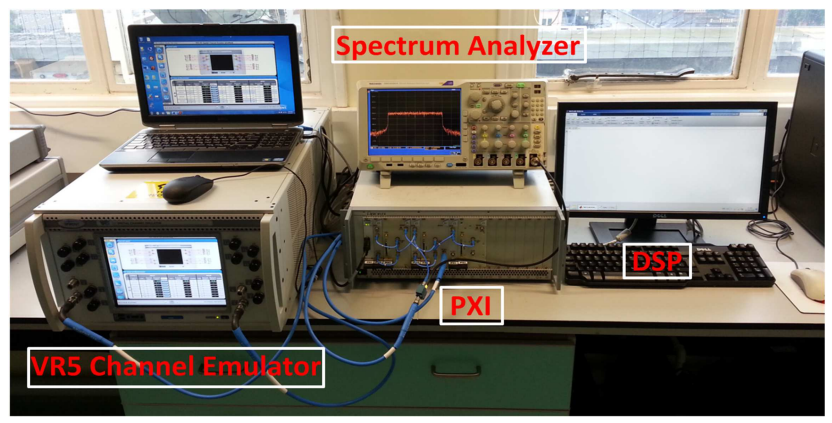

Figure 5. Demonstration of the real experimental setup.

\section{A. Testbed Software}

Signal processing in the DSP blocks IS carried out offline using MATLAB. This part includes operations that generate and recover signals. A block diagram of the software testbed is shown in Fig. 6.

At the transmitter, a simple convolutional coding scheme is used for all the LTE-like experiments (OFDM and SEFDM) to allow fair comparisons. Therefore, the performance obtained is not optimized for each case and improved performance may be obtained if bespoke coding was designed for each case as suggested in [41]. The binary bit stream $\Upsilon=\left[\gamma_{1}, \gamma_{2}, \ldots, \gamma_{\varepsilon}\right]$ is first encoded in the encoder where a coding rate $R_{\text {code }}=1 / 2$. The code used is a $(7,5)$ recursive systematic convolutional (RSC) code of memory 2 with feedforward polynomial $G_{1}(D)=$ $1+D+D^{2}$ and feedback polynomial $G_{2}(D)=1+D^{2}$ [42]. The bits in the coded vector $W=\left[w_{1}, w_{2}, \ldots, w_{\eta}\right]$ of length $\eta=\varepsilon / R_{\text {code }}$, are interleaved using a random interleaver $\Pi$. The interleaved bits $\tilde{S}$ are mapped to the corresponding symbols $S=\left[s_{1}, s_{2}, \ldots, s_{\Theta}\right]$ of length $\Theta=\eta / \log _{2} O$, where $O=4$ is the constellation cardinality in this experiment. One uncoded pilot symbol is inserted at the beginning of each subframe (i.e. 13 complex coded symbols) and is used to estimate 


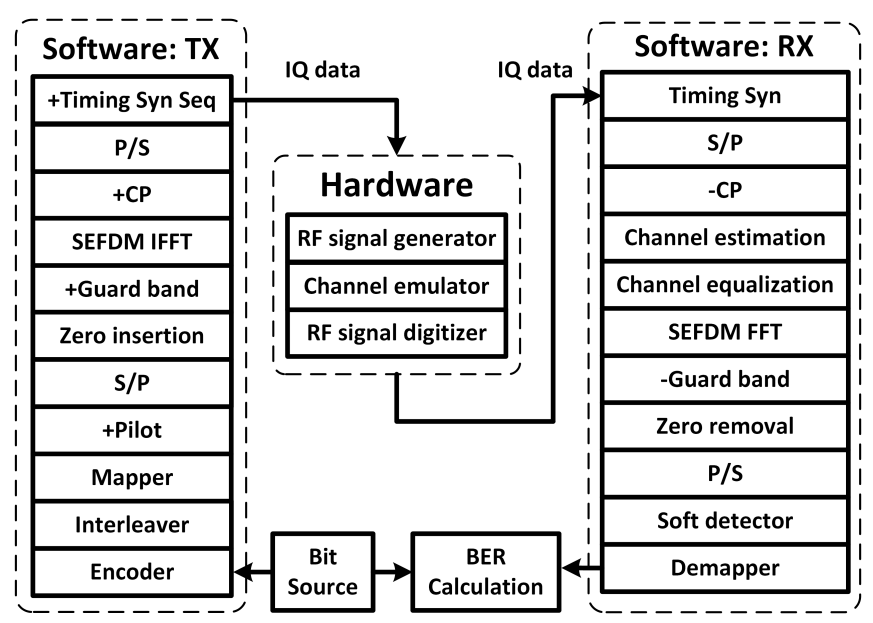

Figure 6. Testbed software processing.

CSI, compensate for imperfect timing synchronization and local oscillator (LO) phase offset. It should be noted that LTE release 8 defines $10 \%$ protection guard sub-carriers. Therefore, we should reserve a $10 \%$ gap between adjacent component carriers to combat the Doppler spread encountered in a real world fading channel. This is managed after a serial to parallel conversion by inserting zeros after the data symbols in each band. The guard band is the sub-carriers which are null for the purpose of oversampling. The modified stream appended with pilot symbols is modulated to specific frequencies by using IFFT operations. In order to combat multipath delay spread, a cyclic prefix (CP) is added before parallel-to-serial (P/S). In order to estimate an accurate starting point of a data stream at receiver side, a timing synchronization sequence is added at the beginning of a transmitted signal. It employs Schmidl and Cox algorithm [43] where two identical data sequence are sent and correlation peak will be calculated and captured at the receiver side. Subsequently, the I and Q data of the SEFDM signal are uploaded to the hardware environment and then captured by the receiver side DSP block.

The captured signal is first timing synchronized and then delivered to a serial to parallel (S/P) transform block. Due to the effects of the multipath fading channel, phase and amplitude distortions are introduced and their impact is estimated and equalized in the channel estimation and channel equalization modules, respectively. The compensated signal is demodulated in the FFT block and raw SEFDM symbols are obtained after the removal of guard bands and inserted zeros. Then the signal detector described in section III-C is used to recover signals from ICI after P/S. Finally, the binary bit stream is obtained after the demapper.

\section{B. Testbed Hardware}

Signal transmission, wireless channel and signal reception are all implemented in a realistic RF environment appeared in Fig. 7. The Aeroflex PXI chassis consists of the 3026C $\mathrm{RF}$ signal generator and the $3035 \mathrm{C}$ RF digitizer. In the RF domain, the Aeroflex $3026 \mathrm{C}$ converts the incoming baseband IQ digital signal to an analogue one and up-converts the

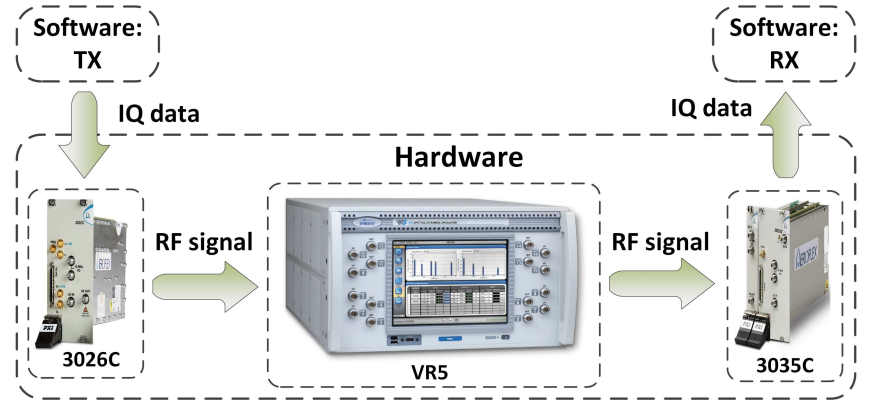

Figure 7. Testbed hardware processing.

analogue signal to a $2 \mathrm{GHz}$ frequency. The RF signal from the $3026 \mathrm{C}$ is transmitted to the input of VR5 whose output is fed to the 3035C. The VR5 passes RF signals through hardware emulated RF channels that can be either user designed of adhere to standard channel models. At the receiver side, after experiencing the fading and time varying channel, the distorted analogue signals are down-converted to baseband and converted back to digital I and Q signals in the Aeroflex 3035C. These signals are captured and then transferred to the RX software block for offline processing. In addition, an external synthesizer termed 3010/3011 RF Synthesizer is used to offer carrier frequency and $10 \mathrm{MHz}$ reference frequency to the 3026C Digital RF Signal Generator and 3035C RF Digitizer.

The Spirent VR5 channel emulator simplifies wireless systems evaluations for technologies such as LTE and LTEAdvanced. It provides a graphical user interface (GUI) for users to set up a real time and complex RF environment. VR5 supports a broad range of frequencies up to $6 \mathrm{GHz}$. There are several simplex (Input or Output only) and duplex (Input/Output) ports in the front panel of the VR5. After selecting the desired connection setup, these connections are automatically made inside the VR5. The propagation conditions like multi-path power delay profile of fading channel models can be edited and saved via the graphical user interface (GUI) and the corresponding multipath effects are reflected in the magnitude of its output spectrum.

Table I

LTE EPA FADING CHANNEL MODEL

\begin{tabular}{|c|c|c|c|}
\hline Path & Doppler frequency & Relative power & Delay values \\
\hline 1 & $5 \mathrm{~Hz}$ & $0.0 \mathrm{~dB}$ & $0 \mathrm{~ns}$ \\
\hline 2 & $5 \mathrm{~Hz}$ & $-1.0 \mathrm{~dB}$ & $30 \mathrm{~ns}$ \\
\hline 3 & $5 \mathrm{~Hz}$ & $-2.0 \mathrm{~dB}$ & $70 \mathrm{~ns}$ \\
\hline 4 & $5 \mathrm{~Hz}$ & $-3.0 \mathrm{~dB}$ & $90 \mathrm{~ns}$ \\
\hline 5 & $5 \mathrm{~Hz}$ & $-8.0 \mathrm{~dB}$ & $110 \mathrm{~ns}$ \\
\hline 6 & $5 \mathrm{~Hz}$ & $-17.2 \mathrm{~dB}$ & $190 \mathrm{~ns}$ \\
\hline 7 & $5 \mathrm{~Hz}$ & $-20.8 \mathrm{~dB}$ & $410 \mathrm{~ns}$ \\
\hline
\end{tabular}

\section{LTE Channel Model}

The third generation partnership project (3GPP) technical recommendation (TR) 36.104 [44] defines three LTE channel models, which are Extended Pedestrian A (EPA), Extended Vehicular A (EVA) and Extended Typical Urban (ETU). The 
experiment in this work was operated using the EPA channel model with detailed information shown in Table I.

Spectral analysis is used to evaluate the frequency response of a signal after propagating through the VR5 channel emulator. Spectra before and after the LTE EPA5 fading channel are shown in Fig. 8.

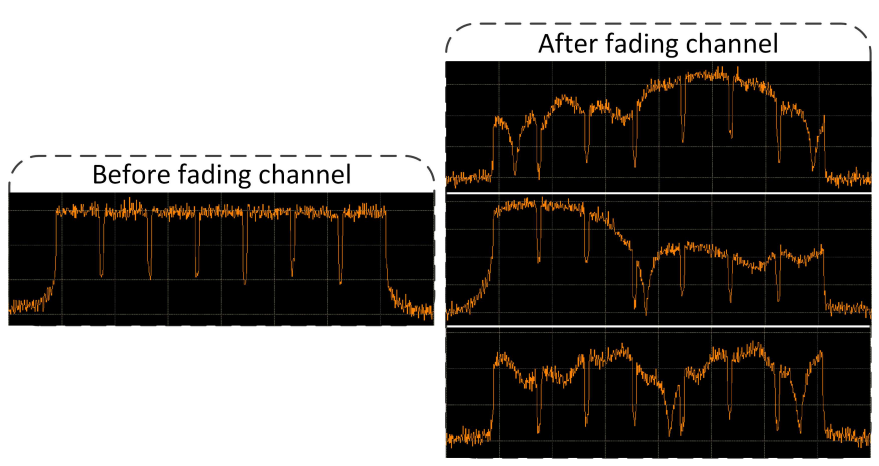

Figure 8. Spectra before and after the LTE EPA5 fading channel.

Spectra shown at three randomly chosen time instants obtained to illustrate the time-variant fading channel characteristics. The spectrum of an CA-SEFDM transmitted signal (without multipath fading) is shown in the left inset of Fig. 8. It is apparent that the spectrum magnitude of the signal is flat across the whole $25 \mathrm{MHz}$ band. After passing through the fading channel, due to random amplitude and phase distortions, the channel responses at different frequencies vary significantly in time as shown in the right inset of Fig. 8. This can be effectively compensated for by using channel estimation and equalization.

\section{EXPERIMENTAL RESULTS}

\section{A. Experimental Conditions}

Table II

EXPERIMENTAL SYSTEM SPECIFICATIONS

\begin{tabular}{ll}
\hline Parameters & Values \\
\hline \hline Central carrier frequency & $2 \mathrm{GHz}$ \\
\hline Sampling frequency & $61.44 \mathrm{MHz}$ \\
\hline CA channel bandwidth & $25 \mathrm{MHz}$ \\
\hline Values of $\alpha$ & $1(\mathrm{OFDM}) ; 0.84 ; 0.72$ \\
\hline Number of CCs & $5 ; 6 ; 7$ \\
\hline Maximum effective bit rate & $20.1 \mathrm{Mbps} ; 23.5 \mathrm{Mbps} ; 27.4 \mathrm{Mbps}$ \\
\hline Bandwidth of one CC & $\alpha \times 5 \mathrm{MHz}$ \\
\hline Sub-carrier baseband bandwidth & $15 \mathrm{KHz}$ \\
\hline Sub-carrier spacing & $\alpha \times 15 \mathrm{KHz}$ \\
\hline IFFT/FFT size & 4096 \\
\hline Occupied sub-carriers in one CC & 301 \\
\hline Cyclic prefix & 288 \\
\hline Modulation scheme & $4 \mathrm{QAM}$ \\
\hline Channel coding & $(7,5)$ convolutional code \\
\hline Coding rate & $R_{\text {code }}=1 / 2$ \\
\hline
\end{tabular}

This experiment is carried out based on the setup shown in section IV. A larger IFFT has to be used since the signal generation for multiple CCs is realized using a single long IFFT following the principle in [35]. System specifications of the experimental testbed are given in Table II. Unlike the maximum bandwidth (100 MHz) defined in LTE-Advanced, a total of $25 \mathrm{MHz}$ bandwidth is used in this experiment. Therefore, the bandwidth for each $\mathrm{CC}$ in a CA-OFDM scenario is $5 \mathrm{MHz}$ while it is $\alpha \times 5 \mathrm{MHz}$ for each $\mathrm{CC}$ in a CASEFDM scenario. The central carrier frequency is set to be $2 \mathrm{GHz}$. Carrier frequencies of other CCs can be obtained by shifting $\alpha \times 5 \mathrm{MHz}$ from the central carrier frequency. A real-time LTE fading channel is configured with the use of the VR5 channel emulator. The channel specifications are shown in Table I. Considering the fact that different SEFDM symbols could experience different channels, which would have different amplitude and phase distortions, a total of 6,500 SEFDM symbols are delivered and measured to get an averaged performance result.

\section{B. Error Performance}

The iteration performance for three systems is studied in Fig. 9 where up to three iterations are tested. In terms of the CA-OFDM system with $5 \mathrm{CCs}$, it is evident that no iteration is required to get converged performance. However, for the CA-SEFDM system with $6 \mathrm{CCs}$, with one iteration, the performance can be slightly improved. Although the improvement is not obvious, in the following BER and effective spectral efficiency demonstrations, one iteration is used for the $6 \mathrm{CCs}$ scenario. The second CA-SEFDM system employs $7 \mathrm{CCs}$ indicating higher interference. As is shown in the first inset of Fig. 9, at least one iteration has to be used in the experiment to get converged performance.

The measured bit error rate is illustrated in Fig. 10. A typical CA-OFDM with $5 \mathrm{CCs}$ is included for the purpose of comparison. The first CA-SEFDM system, aggregating 6 $\mathrm{CCs}$, shows performance close (within $1.7 \mathrm{~dB}$ ) to the CAOFDM one with $16 \%=(1-0.84) \times 100 \%$ bandwidth saving. The second CA-SEFDM system packs sub-carriers even closer by compressing $28 \%=(1-0.72) \times 100 \%$ of bandwidth. Higher bandwidth compression indicates higher interference. Therefore, the performance gap is experimentally shown to be below $3 \mathrm{~dB}$ for all $E_{b} / N_{o}$ values tested.

\section{Spectral Efficiency}

Although CA-SEFDM introduces slight performance degradation, Fig. 11 shows that CA-SEFDM outperforms CAOFDM in terms of effective spectral efficiency, which is defined as the non-error bits per second per Hertz that can be achieved. In Fig. 11, spectral efficiencies are plotted for three different CA schemes at different $E_{b} / N_{o}$ values. The effective spectral efficiency is defined as follows:

$$
\begin{gathered}
R_{a}=(1-B E R) \times R_{\text {code }} \times B_{(C C, \text { OF DM })} \times N_{C C} \times \log _{2} O \\
B=B_{(C C, \text { OF DM })} \times N_{(C C, \text { OF } D M)} \\
S E=R_{a} / B
\end{gathered}
$$

where $R_{a}$ is transmission data rate, $B$ is occupied bandwidth, $S E$ is computed spectral efficiency, $B E R$ is the bit error rate at a specific $E_{b} / N_{o}$ value, $(1-B E R)$ indicates the probability of a non-error received bit stream, $B_{(C C, O F D M)}$ 

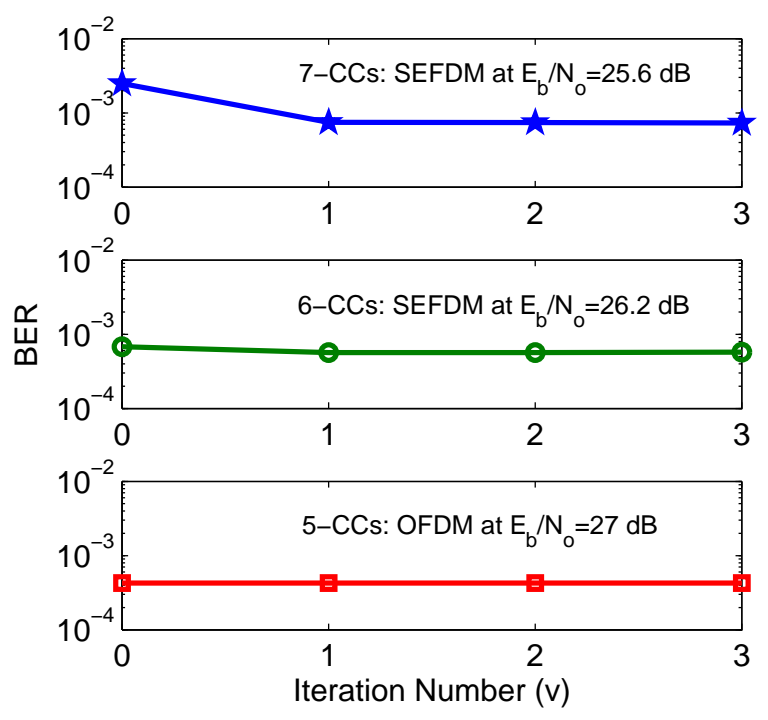

Figure 9. Iteration performance for three systems in the experiment environment.

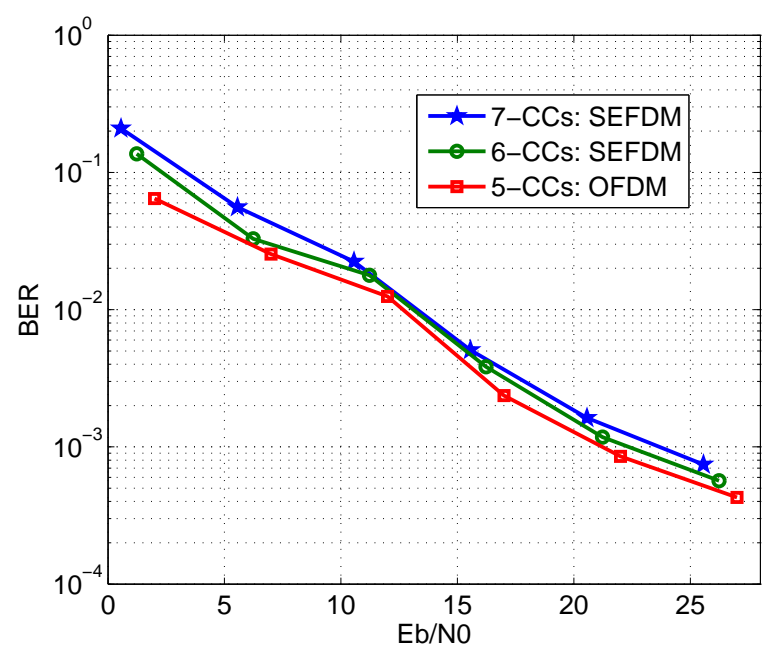

Figure 10. Performance of different CA-SEFDM systems operating in a real RF environment with the LTE EPA fading channel.

is the bandwidth of one CC in OFDM, $N_{C C}$ is the number of CCs in either OFDM or SEFDM, $N_{(C C, O F D M)}$ is the number of CCs in OFDM and $O$ is the constellation cardinality. Fig. 11 shows clearly that CA-SEFDM has a higher effective spectral efficiency than CA-OFDM due to more aggregated CCs in a given bandwidth.

\section{ImPlementation Challenges of the Soft DETECTOR}

To simplify the following discussion, assume an oversampling factor $\rho=1$ resulting in $Q=N$. In terms of a conventional CA-OFDM system, its experimental setup follows the same architecture as illustrated in Fig. 6 except that some modules are specially designed for CA-SEFDM. Firstly, due to the non-orthogonal packed sub-carriers in SEFDM, a typical

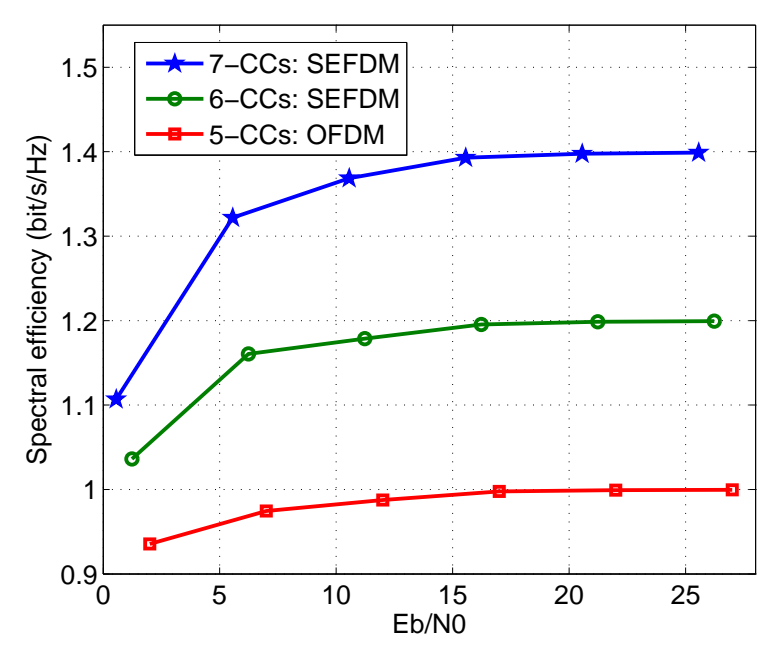

Figure 11. Effective spectral efficiency (bit/s/Hz) of different CA-SEFDM systems computed based on the BER information in Fig. 10 and system specifications in Table II.

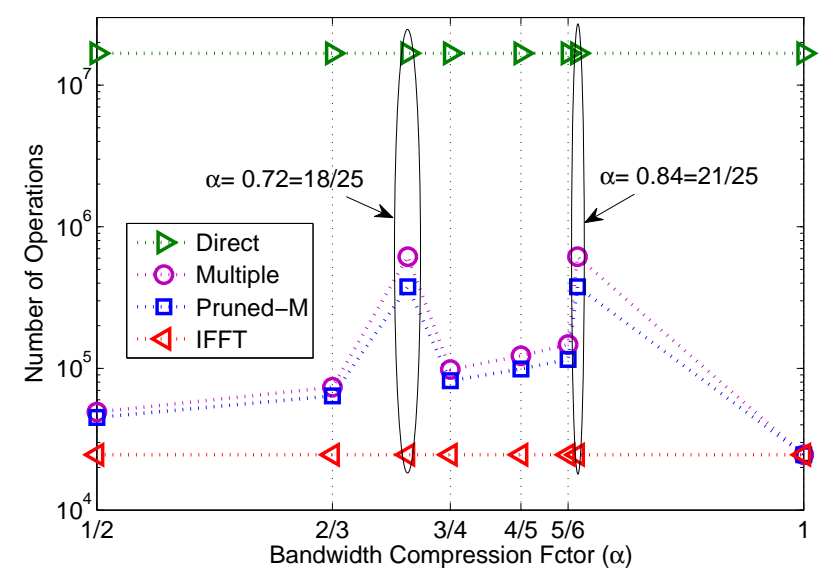

Figure 12. Computational complexity in terms of complex multiplication operations for different algorithms.

IFFT based modulation or FFT based demodulation cannot be used directly. Moreover, in order to mitigate both selfcreated ICI and the ICI from multipath fading, a time-domain channel estimation/equation and a soft detector are introduced specifically for SEFDM.

In typical OFDM systems, the IFFT and FFT are, respectively, efficient transmitter and receiver methods, of similar complexity. For the non-orthogonal SEFDM signals modified algorithms are needed for the purposes of signal generation and detection and these require increased complexity. We show the complexity of different signal generation algorithms using IFFT operations in Table III. The complexity of the converse FFT operations of the receiver, which are parts of the FFT detector of Fig. 4, is calculated in a similar manner.

A direct signal generation technique (applied to any $\alpha$ ) according to (2) is shown in the third column with a higher complexity than the IFFT one. Furthermore, in the next 
Table III

COMPLEXITY IN TERMS OF THE NUMBER OF COMPLEX OPERATIONS FOR DIFFERENT ALGORITHMS.

\begin{tabular}{|c|c|c|c|c|c|c|}
\hline Operations & IFFT(OFDM) & Direct & Single & Multiple & Pruned - S & Pruned - M \\
\hline Multiplications & $\frac{N}{2} \times \log _{2} N$ & $N^{2}$ & $\frac{N}{2 \alpha} \times \log _{2} \frac{N}{\alpha}$ & $c \times\left(\frac{N}{2} \times \log _{2} N\right)$ & $\frac{N}{2 \alpha} \times \log _{2} N$ & $c \times\left(\frac{N}{2} \times \log _{2} \frac{N}{c}\right)$ \\
\hline Additions & $N \times \log _{2} N$ & $N \times(N-1)$ & $\frac{N}{\alpha} \times \log _{2} \frac{N}{\alpha}$ & $c \times\left(N \times \log _{2} N\right)$ & $\frac{N}{\alpha} \times \log _{2} N$ & $c \times\left(N \times \log _{2} \frac{N}{c}\right)$ \\
\hline
\end{tabular}

column, a single IFFT of $N / \alpha$ length is shown with reduced complexity. Subsequently, a multiple IFFT based algorithm including $c$ parallel IFFT blocks each of $N$ points shows its competitive computational complexity. A more efficient algorithm termed 'pruned' IFFT operation was specially investigated for SEFDM in [36] where redundant operations like zero padding or zero insertion are skipped. The 'Pruned$S$ ' indicates the pruned version of the single IFFT while the 'Pruned-M' indicates the multiple IFFTs one. It is apparent that the complexities of all the IFFT based SEFDM algorithms are dependent on both the number of sub-carriers $N$ and the value of $\alpha$.

Fig. 12 shows the computational complexity in terms of complex multiplication operations of several algorithms as a function of the bandwidth compression factor $\alpha$. Generally, the IFFT approach has a competitive advantage since it requires the least operations compared with other methods. The direct algorithm requires a significantly higher number of operations that is more than two orders of magnitude higher than the IFFT one. All IFFT based SEFDM algorithms exhibit a significant reduction in the number of operations compared to the direct method. It should be mentioned that the computational complexities of the IFFT based algorithms vary on the basis of $\alpha$, while the direct approach is independent of $\alpha$. Due to the requirement of the values of $N / \alpha \in 2^{(\mathbb{N}>0)}$ in the single IFFT scheme, its practical use is prohibitive. Therefore, the analysis of its complexity is not included in Fig. 12. It is evident that the computational complexity of the multiple IFFTs algorithm increases with the bandwidth compression factor. This is attributed to the fact that the denominator (i.e. $c$ ) of the bandwidth compression factor $\alpha$ on the x-axis increases. It is also illustrated in Table III that the complexity is proportional to the value of $c$. Therefore, it is inferred that the multiple scheme is applicable to a bandwidth compression factor with small denominators (i.e. c). In Fig. 12, in terms of the multiple IFFT based algorithms including the pruned and non-pruned versions, two peaks are displayed. This is due to the large denominator (i.e. $c=25$ ) of the bandwidth compression factors. In our experiment, in order to demonstrate the idea of CA-SEFDM, the bandwidth compression factors are set to be 0.72 and 0.84 to satisfy the requirements of 7 and 6 aggregated CCs. In practice, the bandwidth compression factors can be selected around 0.72 and 0.84 since adjacent values in Fig. 12 show much lower complexity. In addition, the pruned version of the multiple IFFTs algorithm shows little benefit in complexity reduction compared to the non-pruned version.

Overall, although there are differences in the implementation of CA-OFDM and CA-SEFDM, these are limited. A key difference is in SEFDM's unique and specially designed soft detector, which is used to remove interference with internal structure illustrated in Fig. 4. For both systems, forward error correction (FEC) is required to minimize bit errors and thus the use of BCJR decoder (or equivalent) is common to both systems. Interleaving is an operation to improve the performance of error correcting codes (ECC) by permutating a bit stream to avoid burst errors (e.g. caused by deep fading). At the receiver, a reverse operation termed deinterleaving $\Pi^{-1}$ is required. In SEFDM, an additional interleaver is required in the soft detector for the iterative process. Clearly, the interleaver $\Pi$ within the SEFDM soft detector necessitates additional resource consumption. The complexity of the FFT detector may be deduced from Table III since the basic operations within the FFT detector are FFT and IFFT operations of similar complexity [31].

\section{CONCLUSIONS}

This work introduces a new framework to increase the number of component carriers (CCs), in a carrier aggregation (CA) system, within a limited bandwidth and without changing the transmission rate per sub-carrier, by using bandwidth compressed spectrally efficient FDM (SEFDM) signals. Results illustrate that overall bit rate can be increased whilst maintaining the same bandwidth. Using signal structure based on LTE-Advanced and through some modification of the pilot symbol structure, this work shows experimentally that compressing the sub-carrier spacing within each $\mathrm{CC}$ by $16 \%$, a CA-SEFDM system can integrate 6 CCs into the same 25 $\mathrm{MHz}$ bandwidth used for a CA-OFDM system with $5 \mathrm{CCs}$. Moreover, with further compression by $28 \%$, one more CC can be aggregated. The superiority of CA-SEFDM is demonstrated by comparing it to CA-OFDM.

This experimental work showed the feasibility of increasing bit rate by up to $40 \%$ at the expense of $3 \mathrm{~dB}$ increase in power penalty and increased implementation complexity, especially at the receiver. Such experimental results were obtained by testing signals over an LTE channel generated using a commercially available channel emulator operating in the $2 \mathrm{GHz}$ band. If signals proposed here were to replace the LTE standard signals, clearly compatibility issues have to be addressed. Although the bandwidth and spectral allocation are the same, changes in the number of component carriers and sub-carrier frequency spacing as well as the required changes for pilot tone arrangements would require changes to existing LTE standards. This may be a reasonable price to incur given the bandwidth saving advantages.

\section{REFERENCES}

[1] T. Xu and I. Darwazeh, "Bandwidth compressed carrier aggregation," in IEEE ICC 2015 - Workshop on $5 G$ \& Beyond - Enabling Technologies 
and Applications (ICC'15 - Workshops 23), London, United Kingdom, Jun. 2015, pp. 1107-1112.

[2] 3GPP TS 36.300 version 8.12.0 Release 8, "Evolved universal terrestrial radio access (E-UTRA) and evolved universal terrestrial radio access network (E-UTRAN); overall description; stage 2 (release 8)," April 2010.

[3] 3GPP TR 36.912 v.13.0.0, "Feasibility study for further advancements for E-UTRA (LTE-Advanced)," Rel. 13, Dec. 2015.

[4] M. Iwamura, K. Etemad, M.-H. Fong, R. Nory, and R. Love, "Carrier aggregation framework in 3GPP LTE-advanced [WiMAX/LTE update]," Communications Magazine, IEEE, vol. 48, no. 8, pp. 60-67, August 2010.

[5] K. Pedersen, F. Frederiksen, C. Rosa, H. Nguyen, L. Garcia, and Y. Wang, "Carrier aggregation for LTE-advanced: functionality and performance aspects," Communications Magazine, IEEE, vol. 49, no. 6, pp. 89-95, June 2011.

[6] D. Bai, C. Park, J. Lee, H. Nguyen, J. Singh, A. Gupta, Z. Pi, T. Kim, C. Lim, M.-G. Kim, and I. Kang, "LTE-advanced modem design: challenges and perspectives," Communications Magazine, IEEE, vol. 50, no. 2, pp. 178-186, February 2012.

[7] C. Park, L. Sundstrom, A. Wallen, and A. Khayrallah, "Carrier aggregation for LTE-advanced: design challenges of terminals," Communications Magazine, IEEE, vol. 51, no. 12, pp. 76-84, December 2013.

[8] M. Rodrigues and I. Darwazeh, "A spectrally efficient frequency division multiplexing based communications system," in Proc. 8th Int. OFDM Workshop, Hamburg, 2003, pp. 48-49.

[9] T. Xu and I. Darwazeh, "Spectrally efficient FDM: Spectrum saving technique for 5G?" in $5 G$ for Ubiquitous Connectivity (5GU), 20141 st International Conference on, Nov 2014, pp. 273-278.

[10] I. Darwazeh, T. Xu, T. Gui, Y. Bao, and Z. Li, "Optical SEFDM system; bandwidth saving using non-orthogonal sub-carriers," Photonics Technology Letters, IEEE, vol. 26, no. 4, pp. 352-355, Feb 2014.

[11] D. Nopchinda, T. Xu, R. Maher, B. Thomsen, and I. Darwazeh, "Dual polarization coherent optical spectrally efficient frequency division multiplexing," Photonics Technology Letters, IEEE, vol. 28, no. 1, pp. 83-86, Jan 2016.

[12] T. Xu, S. Mikroulis, J. E. Mitchell, and I. Darwazeh, "Bandwidth compressed waveform for $60-\mathrm{GHz}$ millimeter-wave radio over fiber experiment," Journal of Lightwave Technology, vol. 34, no. 14, pp. 3458-3465, July 2016.

[13] F.-L. Luo and C. J. Zhang, Signal Processing for 5G: Algorithms and Implementations. Wiley, 2016.

[14] J. Andrews, S. Buzzi, W. Choi, S. Hanly, A. Lozano, A. Soong, and J. Zhang, "What will 5G be?" Selected Areas in Communications, IEEE Journal on, vol. 32, no. 6, pp. 1065-1082, June 2014.

[15] J. Anderson, F. Rusek, and V. Öwall, "Faster-than-Nyquist signaling," Proceedings of the IEEE, vol. 101, no. 8, pp. 1817-1830, 2013.

[16] J. Mazo, "Faster-than-Nyquist signaling," Bell Syst. Tech. J, vol. 54, no. 8, pp. 1451-1462, 1975.

[17] F. Rusek and J. Anderson, "The two dimensional Mazo limit," in Information Theory, 2005. ISIT 2005. Proceedings. International Symposium on, Sept 2005, pp. 970-974.

[18] _ " "Multistream faster than Nyquist signaling," Communications, IEEE Transactions on, vol. 57, no. 5, pp. 1329-1340, May 2009.

[19] D. Dasalukunte, F. Rusek, and V. Öwall, "An 0.8- $\mathrm{mm}^{2}$ 9.6- $m w$ iterative decoder for faster-than-Nyquist and orthogonal signaling multicarrier systems in 65-nm CMOS," Solid-State Circuits, IEEE Journal of, vol. 48, no. 7, pp. 1680-1688, July 2013.

[20] A. Barbieri, D. Fertonani, and G. Colavolpe, "Time-frequency packing for linear modulations: spectral efficiency and practical detection schemes," Communications, IEEE Transactions on, vol. 57, no. 10, pp. 2951-2959, October 2009.

[21] B. Farhang-Boroujeny, "OFDM versus filter bank multicarrier," Signal Processing Magazine, IEEE, vol. 28, no. 3, pp. 92-112, May 2011.

[22] G. Fettweis, M. Krondorf, and S. Bittner, "GFDM - generalized frequency division multiplexing," in Vehicular Technology Conference, 2009. VTC Spring 2009. IEEE 69th, April 2009, pp. 1-4.

[23] V. Vakilian, T. Wild, F. Schaich, S. Ten Brink, and J.-F. Frigon, "Universal-filtered multi-carrier technique for wireless systems beyond LTE," in Globecom Workshops (GC Wkshps), 2013 IEEE, Dec 2013, pp. 223-228.

[24] D. Rainnie, Y. Feng, and J. Bajcsy, "On capacity merits of spectrally efficient FDM," in Military Communications Conference, MILCOM 2015 - 2015 IEEE, Oct 2015, pp. 581-586.

[25] S. V. Zavjalov, S. B. Makarov, S. V. Volvenko, and W. Xue, "Waveform optimization of SEFDM signals with constraints on bandwidth and an out-of-band emission level," in Internet of Things, Smart Spaces, and
Next Generation Networks and Systems - 15th International Conference, NEW2AN 2015, and 8th Conference, ruSMART 2015, St. Petersburg, Russia, August 26-28, 2015, Proceedings, 2015, pp. 636-646.

[26] T. Xu and I. Darwazeh, "Nyquist-SEFDM: pulse shaped multicarrier communication with Sub-Carrier spacing below the symbol rate," in 2016 10th International Symposium on Communication Systems, Networks and Digital Signal Processing (CSNDSP) (CSNDSP16), Prague, Czech Republic, Jul. 2016.

[27] S. Isam, I. Kanaras, and I. Darwazeh, "A truncated SVD approach for fixed complexity spectrally efficient FDM receivers," in Wireless Communications and Networking Conference (WCNC), 2011 IEEE, March 2011, pp. 1584-1589.

[28] I. Kanaras, A. Chorti, M. Rodrigues, and I. Darwazeh, "A fast constrained sphere decoder for ill conditioned communication systems," Communications Letters, IEEE, vol. 14, no. 11, pp. 999-1001, November 2010.

[29] T. Xu, R. C. Grammenos, F. Marvasti, and I. Darwazeh, "An improved fixed sphere decoder employing soft decision for the detection of nonorthogonal signals," Communications Letters, IEEE, vol. 17, no. 10, pp. 1964-1967, October 2013.

[30] T. Xu and I. Darwazeh, "Multi-Band reduced complexity spectrally efficient FDM systems," in 9th IEEE/IET International Symposium on Communication Systems, Networks \& Digital Signal Processing 2014 (CSNDSP14), Manchester, United Kingdom, Jul. 2014, pp. 904-909.

[31] _ , "A soft detector for spectrally efficient systems with nonorthogonal overlapped sub-carriers," Communications Letters, IEEE, vol. 18 , no. 10, pp. 1847-1850, Oct 2014.

[32] L. Bahl, J. Cocke, F. Jelinek, and J. Raviv, "Optimal decoding of linear codes for minimizing symbol error rate (Corresp.)," Information Theory, IEEE Transactions on, vol. 20, no. 2, pp. 284-287, Mar 1974.

[33] D. K. Fadeev and A. V. Rashich, "Optimal input power backoff of a nonlinear power amplifier for SEFDM system," in Internet of Things, Smart Spaces, and Next Generation Networks and Systems - 15th International Conference, NEW2AN 2015, and 8th Conference, ruSMART 2015, St. Petersburg, Russia, August 26-28, 2015, Proceedings, 2015, pp. 669-678.

[34] V. Rashich and A. Rashich, "Asymptotically optimal algorithm for OFDM-signal reception under AWGN and OFDM interference shifted in frequency," in Communications and Networking (BlackSeaCom), 2015 IEEE International Black Sea Conference on, May 2015, pp. 5-8.

[35] S. Bassam, W. Chen, M. Helaoui, and F. Ghannouchi, "Transmitter architecture for CA: carrier aggregation in LTE-advanced systems," Microwave Magazine, IEEE, vol. 14, no. 5, pp. 78-86, July 2013.

[36] P. Whatmough, M. Perrett, S. Isam, and I. Darwazeh, "VLSI architecture for a reconfigurable spectrally efficient FDM baseband transmitter," Circuits and Systems I: Regular Papers, IEEE Transactions on, vol. 59, no. 5, pp. 1107-1118, May 2012.

[37] A. Chorti, I. Kanaras, M. Rodrigues, and I. Darwazeh, "Joint channel equalization and detection of spectrally efficient FDM signals," in Personal Indoor and Mobile Radio Communications (PIMRC), 2010 IEEE 21st International Symposium on, Sept 2010, pp. 177-182.

[38] S. Isam and I. Darwazeh, "Robust channel estimation for spectrally efficient FDM system," in Telecommunications (ICT), 2012 19th International Conference on, April 2012, pp. 1-6.

[39] J. B. Anderson and F. Rusek, "Improving OFDM: Multistream fasterthan-Nyquist signaling," in Turbo Codes Related Topics; 6th International ITG-Conference on Source and Channel Coding (TURBOCOD$I N G), 2006$ 4th International Symposium on, April 2006, pp. 1-5.

[40] J. Hagenauer, "The turbo principle: tutorial introduction and state of the art," in Proc. Int. Symp. Turbo Codes, Sept 1997, pp. 1-11.

[41] P. Banelli, S. Buzzi, G. Colavolpe, A. Modenini, F. Rusek, and A. Ugolini, "Modulation formats and waveforms for $5 \mathrm{G}$ networks: Who will be the heir of OFDM?: An overview of alternative modulation schemes for improved spectral efficiency," Signal Processing Magazine, IEEE, vol. 31, no. 6, pp. 80-93, Nov 2014.

[42] B. Hochwald and S. Ten Brink, "Achieving near-capacity on a multipleantenna channel," Communications, IEEE Transactions on, vol. 51, no. 3, pp. 389-399, March 2003.

[43] T. Schmidl and D. Cox, "Robust frequency and timing synchronization for OFDM," Communications, IEEE Transactions on, vol. 45, no. 12, pp. 1613-1621, Dec 1997.

[44] 3GPP Evolved Universal Terrestrial Radio Access (E-UTRA) TS 36.104 V10.2.0, "Base station (BS) radio transmission and reception," May 2011. 


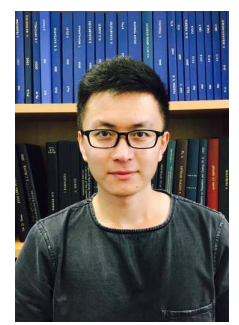

Tongyang Xu (S'13) received his B.Sc. degree in electronic information engineering from Xidian University, Xi' an, China, in 2011, and M.Sc. degree (with distinction) in Telecommunications from University College London (UCL), London, U.K., in 2012. He is currently working toward his Ph.D. degree at UCL.

In 2013, he joined the Communications and Information Systems group, Department of Electronic and Electrical Engineering, University College London. His research interests include future 5th generation (5G) communications technology, waveform design, spectral efficiency improvement, and real-time testbed design. His current research work concerns the design and implementation of optical and wireless spectrally efficient communication systems on real-time experimental platforms.

$\mathrm{Mr}$. Xu was the recipient of the Faculty of Engineering Sciences Scholarship at UCL in 2013.

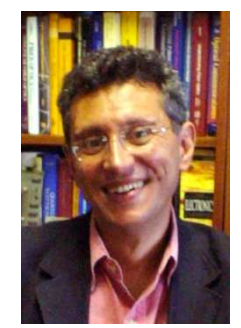

Izzat Darwazeh (SM'03) currently holds the University of London Chair of Communications Engineering and leads the 70-strong Communications and Information Systems Group of the Department of Electronic and Electrical Engineering, University College London (UCL) in the U.K. He is a graduate of the University of Jordan (Electrical Engineering 1984) and has obtained the MSc (1986) and a PhD (1991) degrees from the University of Manchester in the UK.

He has authored or coauthored more than 250 papers in the areas of optical and wireless communications and monolithic microwave integrated circuits (MMICs) and high-speed/frequency circuits. He coedited Analogue Optical Fibre Communications (IEE, 1995) and was a co-editor and of the 2008 Elsevier-Newness book on Electrical Engineering. $\mathrm{He}$ is also the co-author of two books; the 2005 book on Linear Circuit Analysis and Modelling (Elsevier) and of the 2015 book on Microwave Active Circuit Analysis and Design (Academic Press). He currently teaches mobile and wireless communications and circuit design and his current research activities are in ultra high-speed microwave circuits and in wireless and optical communication systems. In 2003 he proposed (with Miguel Rodrigues) the SEFDM concept and has been working in this topic since then. Prof. Darwazeh is a Chartered Engineer, Fellow of the IET and a Fellow of the Institute of Telecommunications Professionals FITP. 